\title{
Effect of Novel Quick Freezing Techniques Combined with Different Thawing Processes on Beef Quality
}

\author{
Yeon-Ji Jo, Min-Young Jang, You-Kyoung Jung, Jae-Hyeong Kim, Jun-Bo Sim, Ji-Yeon Chun, \\ Seon-Mi Yoo ${ }^{1}$, Gui-Jung Han ${ }^{1}$, and Sang-Gi Min* \\ Department of Bioindustrial Technologies, Konkuk University, Seoul 143-701, Korea \\ ${ }^{1}$ National Academy of Agricultural Science, Rural Development Administration, Jeonju 565-851, Korea
}

\begin{abstract}
This study investigated the effect of various freezing and thawing techniques on the quality of beef. Meat samples were frozen using natural convection freezing (NF), individual quick freezing (IQF), or cryogenic freezing (CF) techniques, followed by natural convection thawing (NCT) or running water thawing (RT). The meat was frozen until the core temperature reached $-12^{\circ} \mathrm{C}$ and then stored at $-24^{\circ} \mathrm{C}$, followed by thawing until the temperature reached $5^{\circ} \mathrm{C}$. Quality parameters, such as the $\mathrm{pH}$, water binding properties, CIE color, shear force, and microstructure of the beef were elucidated. Although the freezing and thawing combinations did not cause remarkable changes in the quality parameters, rapid freezing, in the order of CF, IQF, and NF, was found to minimize the quality deterioration. In the case of thawing methods, NCT was better than RT and the meat quality was influence on the thawing temperature rather than the thawing rate. Although the microstructure of the frozen beef exhibited an excessive loss of integrity after the freezing and thawing, it did not cause any remarkable change in the beef quality. Taken together, these results demonstrate that $\mathrm{CF}$ and NCT form the best combination for beef processing; however, IQF and NCT may have practical applications in the frozen food industry.
\end{abstract}

Keywords: quick freezing, cryogenic, thawing, beef, quality

\section{Introduction}

Freezing is the best method for food preservation. Water undergoes phase transition at subzero temperatures leading to ice crystal formation. Tissue damage in frozen foods inevitably occurs due to the differential density of ice and water. Upon thawing, the physically damaged tissue liberates moisture (drip loss), resulting in the loss of its nutritional value and quality. To minimize this tissue damage, rapid freezing techniques are strongly recommended (Hong et al., 2005).

The tissue deterioration caused by ice crystal formation depends on the number and size of the ice crystals. The former relies on the extent of supercooling, while the latter is directly proportional to the phase transition time (Fernández et al., 2006). To achieve rapid freezing, therefore, greater supercooling with shorter phase transition times is necessary. Although several novel freezing tech-

*Corresponding author: Sang-Gi Min, Department of Bioindus trial Technologies, Konkuk University, Seoul 143-701, Korea. Tel: +82-2-450-3680, Fax: +82-2-455-1044, E-mail: minsg@ konkuk.ac.kr niques including dehydrofreezing, cryogenic freezing (CF), as well as high pressure freezing (Li and Sun, 2002) have been developed, they have limited applications in the frozen food industry due to technical problems and cost-inefficiency (Hong et al., 2009).

Individual quick freezing (IQF) is an advanced form of classical air blast freezing. In IQF, diced or sliced food samples are frozen in an air blast freezer at relatively lower temperatures $\left(-30\right.$ to $\left.-40^{\circ} \mathrm{C}\right)$, which enables ultrafast freezing, compared to conventional freezing. In the case of vegetables and fruits, it is also possible to greatly reduce the overall freezing time.

The importance of the thawing process should not be neglected while evaluating the quality of frozen foods, because it depends on both, the freezing and thawing procedures. Compared to the extensive research on freezing technology, little information is available on novel thawing techniques. Ice has greater heat conductivity and heat diffusivity than water (Carson, 2006; James, 1968). During freezing, heat trapped in the interior of foods escapes through the frozen surfaces. An alternate medium for heat transfer during thawing is the thawed surface of the frozen foods itself (Leung et al., 2007). Theoretically, the tha- 
wing process takes longer than the freezing process, with respect to the differences in the physical properties of ice and water. Recrystallization during thawing triggers the deterioration of the quality of the frozen foods. Therefore, it is essential to thaw frozen foods rapidly, which can be achieved by applying a high temperature medium. Since running water has a higher heat transfer coefficient than still air (Hu and Sun, 2001), thawing in running water (RT) takes less time compared to natural air convection thawing (NCT).

For practical applications, CF and IQF are potentially advantage to facilitate rapid freezing of foodstuffs. In addition, reducing the dimensions of the food ensures that the application of relatively high temperatures of the thawing medium does not affect the microbial growth. In this study, therefore, effects of various novel freezing techniques combined with two thawing processes, NCT and RT, on the quality of beef samples.

\section{Materials and Methods}

\section{Materials and sample preparation}

Beef (Hanwoo, $1^{++} \mathrm{A}$ grade) eye of rounds were obtained from three carcasses at $48 \mathrm{~h}$ post mortem. All the visible fat and connective tissue was trimmed off, and the beef was cut into a rectangular form $(1 \times 1 \times 5 \mathrm{~cm}, 9 \mathrm{~g})$, parallel to the direction of the muscle fiber. For the fresh (non-frozen) control, parts of the samples were placed at $4^{\circ} \mathrm{C}$ for $24 \mathrm{~h}$. The remaining portions of the meat strips were divided into three groups for treatments. A thermocouple ( $k$-type) was inserted into the geometric center of six samples for each treatment, to monitor the temperature of the samples during freezing and thawing. For reproducibility, the entire experiment was repeated three times on different days $(\mathrm{n}=3)$.

\section{Freezing and thawing procedures}

Natural convection freezing (NF) was conducted at $-24^{\circ} \mathrm{C}$ in a conventional freezer, while IQF was performed using a $-45^{\circ} \mathrm{C}$ air blast freezer (SEOJIN Freezer, Korea). For CF, samples were sprayed in Cryo-chamber system $(150 \times 30 \times 50 \mathrm{~cm}[\mathrm{~L} \times \mathrm{W} \times \mathrm{H}]$, HyunDae FA, Korea) using four circular spray nozzle (MS TECH, Korea) with $60^{\circ}$ of spray angle and $9.0 \mathrm{~L} / \mathrm{min}$ of flow rate, which exposed to liquid nitrogen vapor. The samples were cryogenically frozen $\left(-100^{\circ} \mathrm{C}\right)$ for 2 min $30 \mathrm{sec}$. All freezing treatment was finished when the geometrically core temperature of meat strips was reached at $-12^{\circ} \mathrm{C}$. Each freezing treatment was further divided into two groups, vacuum packaged in polyethylene pouches, and placed in a $-24^{\circ} \mathrm{C}$ conventional freezer for $24 \mathrm{~h}$. For thawing, one group of each freezing treatment was thawed in running water (RT; $10^{\circ} \mathrm{C}$ ) and the other group was kept at $4^{\circ} \mathrm{C}$ for the NCT treatment. Thawing was done when the core temperature reached $5^{\circ} \mathrm{C}$. The temperature profiles of all the treatments were monitored by connecting the thermocouple with a mobile corder (MV-100, Yokogawa Co., Ltd., Japan).

\section{pH}

The $\mathrm{pH}$ of the treated and control samples was measured with a pH meter (S-220, Mettler-Toledo Co., Switzerland). Samples ( $5 \mathrm{~g}$ ) from 3 meat strips were homogenized in $45 \mathrm{~mL}$ distilled water for $1 \mathrm{~min}$ and the $\mathrm{pH}$ of the sample homogenate was determined in triplicate.

\section{Instrumental color}

Color measurement was performed using a colorimeter (CR-400, Konica-Minolta Co., Japan), calibrated with a white standard plate $\left(L^{*}=+97.83, a^{*}=-0.43, b^{*}=+1.98\right)$. CIE $L^{*}, a^{*}$, and $b^{*}$ were determined as the indicators of lightness, redness, and yellowness, respectively. The color was taken from three random places on the beef surface. Total color difference $(\Delta E)$ was numerically calculated using the color difference between the treatments and the control using the following equation:

$$
\Delta E=\sqrt{\left(\Delta L^{*}\right)^{2}+\left(\Delta a^{*}\right)^{2}+\left(\Delta b^{*}\right)^{2}}
$$

\section{Thawing loss and cooking loss}

After the thawing treatment, the beef surface exudate was gently removed using a tissue, and the samples were weighed. Thawing loss was determined by calculating the weight differences before freezing and after thawing. After determining the thawing loss, the samples were bagged in polyethylene pouches and thermally treated in an $80^{\circ} \mathrm{C}$ water bath (DX9, Hanyoung Co., Korea) until the core temperature reached $75^{\circ} \mathrm{C}$. Cooking loss was calculated as the difference in weight before and after cooking. The test was done in triplicate.

\section{Water holding capacity (WHC)}

WHC was determined by the method reported by Hong et al. (2005). Approximately $1 \mathrm{~g}$ of thawed beef was weighed and placed into a centrifuge tube along with gauze as an absorbent. The samples were centrifuged at $1,500 \times g$ for $10 \mathrm{~min}$ at $4^{\circ} \mathrm{C}$ using an automatic refrigerated centrifuge (1736R, LABOGENE, Korea). After centrifuging, the beef was removed from the tube and the weight of the 
centrifuge tubes before and after drying was measured. WHC assessment was done in triplicate and expressed as the percentage of moisture remaining in the meat.

\section{Shear force}

The shear force of the beef samples before and after cooking was determined in triplicate using a Texture Analyzer (CT3, Brookfield Co., USA) equipped with $v$-type plain probe. The conditions of texture analysis were as follows: compression type, test speed of $2.5 \mathrm{~mm} / \mathrm{s}$, target distance of $15 \mathrm{~mm}$, and trigger load of $650 \mathrm{~g}$. The test was repeated at least 16 times.

\section{Light microscopy}

Light microscopy of fresh and frozen beef tissue was conducted on $0.2 \mathrm{~cm}$-thick sections of formalin-fixed, paraffin-embedded samples stained with hematoxylin and eosin (H\&E; BBC Biochemical, USA), using autostainer (Leica autostainer X1 ST5010 Autostainer XL, Leica Microsystems Ltd., Korea).

\section{Statistical analysis}

Completely randomized design was adopted to analyze the data obtained from three independent experiments $(\mathrm{n}=$ $3)$. One-way analysis of variance (ANOVA) was performed on all the variables using the General Linear Model (GLM) procedure (SAS 9.3, SAS Institute, USA). Differences among the means were compared using Tukey's Studentized Range (HSD) Test $(p<0.05)$.

\section{Results and Discussion}

\section{Freezing/thawing times}

NF exhibited the longest freezing time (55 min), followed by $18 \mathrm{~min}$ of IQF, and $3 \mathrm{~min}$ of CF (Fig. 1). The freezing temperature is expected to strongly affect the freezing time of the beef samples. In the present study, the mean temperature of each freezing treatment was -24 , -40 , and $-100^{\circ} \mathrm{C}$ for NF, IQF, and CF, respectively. Since the driving force of the freezing rate is the freezing temperature (Hong et al., 2009), CF and IQF enabled rapid freezing of the beef, compared to NF. A faster rate of thawing was achieved by RT compared to NCT, with a total thawing time of 7-9 min for RT compared to 48-59 min for NCT (Fig. 1). Alternately, IQF and CF exhibited the shortest and the longest thawing times, respectively. Although the overall thawing time for NF was slightly longer than that for IQF, the difference was not statistically significant.
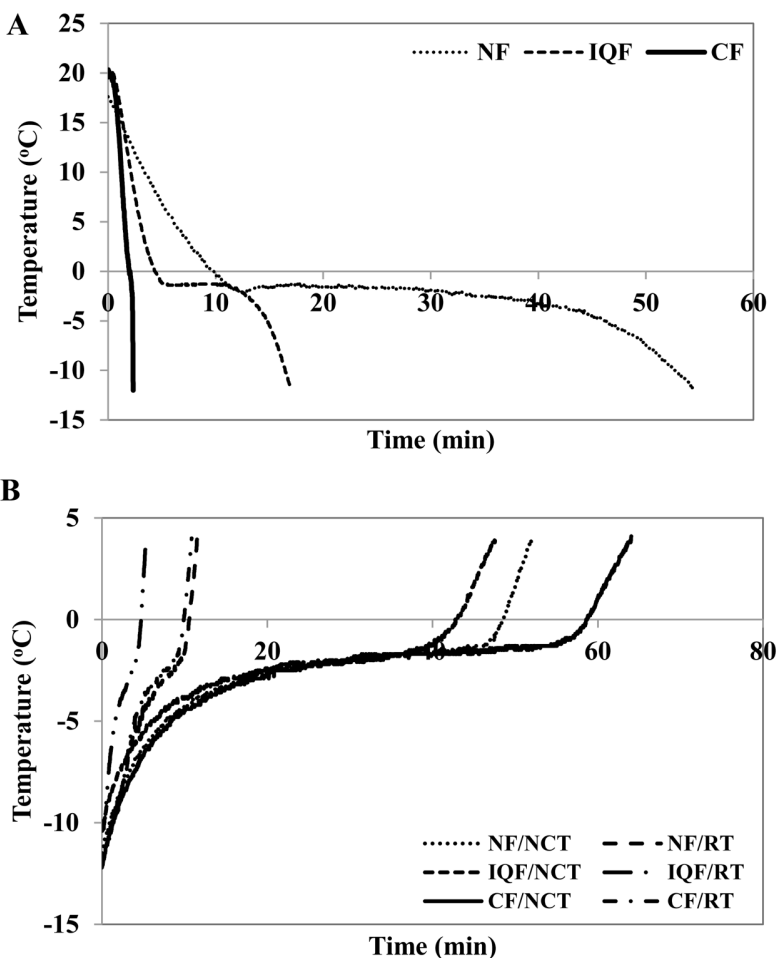

Fig. 1. Time-temperature profiles of the (A) freezing and (B) thawing procedures. The treatments include natural convection freezing (NF), individual quick freezing (IQF), cryogenic freezing (CF), natural convection thawing (NCT), and running water thawing (RT).

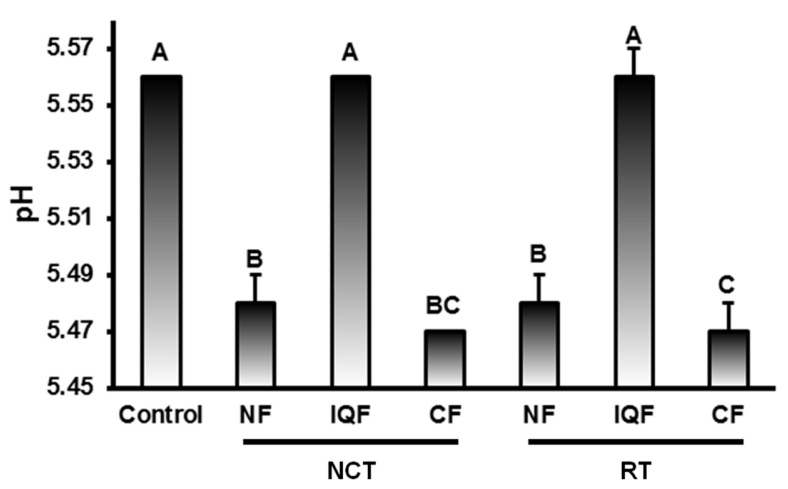

Fig. 2. Effects of the freezing and thawing methods on the pH of beef samples. The error bars indicate standard deviation $(\mathrm{n}=3)$. Bars with different letters are significantly different $(p<0.05)$. The Y-axis denotes treatments including natural convection freezing (NF), individual quick freezing (IQF), cryogenic freezing (CF), natural convection thawing (NCT), and running water thawing (RT).

pH

Initially, the $\mathrm{pH}$ of the fresh control was 5.56 (Fig. 2), which is within the general $\mathrm{pH}$ range of post-rigor beef (Carse and Locker, 1974). Following the freezing and tha- 
wing processes, the $\mathrm{pH}$ of the beef decreased to 5.47-5.48 $(p<0.05)$, with the exception of the IQF treatment, for which the $\mathrm{pH}$ remained unchanged by the freezing/thawing processes. Irrespective of the freezing treatments, there was no significant difference in $\mathrm{pH}$ between the two thawing methods. In general, the $\mathrm{pH}$ of frozen/thawed meat can be higher or lower than that of the fresh control, depending on the freezing and thawing procedures applied. The higher $\mathrm{pH}$ can be attributed to cold denaturation (Meersman et al., 2006), while the lower $\mathrm{pH}$ may be caused by the release of hydrogen ions (Leygonie et al., 2011). Wagner and Aňón (1985) demonstrated that slower freezing of meat leads to increased myofibrillar protein denaturation, causing an increase in the $\mathrm{pH}$, although it should be noted that the authors tried to freeze the extracted myofibrillar protein, which is highly sensitive to temperature stress. Farouk et al. (2004) observed that the freezing rate does not influence the $\mathrm{pH}$ of intact muscle, and concluded that ultra-fast freezing and storage at low temperatures are not necessary to maintain the functional properties of meat. Our previous study (Hong et al., 2005) also showed that the $\mathrm{pH}$ of meat is affected by thawing rather than the freezing process. Thawing is generally achieved by heat transfer from the surface to the interior of frozen foods. It is likely that protein denaturation that occurs during the freezing and thawing cycles is affected by the thawing temperature and not the thawing rate (Leygonie et al., 2012). In the present study, meat was subjected to relatively high temperatures during thawing compared to NCT. Nevertheless, shorter thawing times achieved by RT can minimize the chances of protein unfolding, thereby resulting in no difference in $\mathrm{pH}$ between $\mathrm{RT}$ and NCT.

\section{Water binding properties}

Table 1 shows the effects of the freezing and thawing methods on thawing loss, cooking loss, and WHC of beef. The overall thawing loss of beef was in the range of 2.3-4.5\%. The IQF treatment tended to show a slightly higher thawing loss than NF and CF treatments, possibly due to evaporative loss during freezing. Furthermore, the NCT treatment exhibited slightly higher thawing loss than the RT treatment. However, these differences were not significant, with the exception of the IQF/NCT and CF/ $\mathrm{RT}$ treatments $(p<0.05)$. The lowest thawing loss achieved by the $\mathrm{CF} / \mathrm{RT}$ combination is possibly due to the rapid freezing and thawing treatment. Cooking loss of control beef was $17 \%$, which was not significantly different from that achieved by the treatments. However, the cooking loss caused by IQF and CF with NCT was significantly
Table 1. Effects of freezing and thawing methods on drip loss, cooking loss, and water holding capacity of beef

\begin{tabular}{|c|c|c|c|}
\hline \multirow{2}{*}{$\begin{array}{l}\text { Freezing } \\
\text { system }^{1)}\end{array}$} & \multicolumn{3}{|c|}{ Thawing system ${ }^{21}$} \\
\hline & Control & NCT & RT \\
\hline \multicolumn{4}{|c|}{ Drip loss (\%) } \\
\hline NF & & $3.63 \pm 1.57^{\mathrm{AB}}$ & $3.60 \pm 1.20^{\mathrm{AB}}$ \\
\hline IQF & & $4.46 \pm 0.10^{\mathrm{A}}$ & $3.60 \pm 0.69^{\mathrm{AB}}$ \\
\hline CF & & $2.51 \pm 0.59^{\mathrm{AB}}$ & $2.33 \pm 1.17^{\mathrm{B}}$ \\
\hline \multicolumn{4}{|c|}{ Cooking loss (\%) } \\
\hline NF & & $21.2 \pm 2.00^{\mathrm{AB}}$ & $20.1 \pm 1.74^{\mathrm{AB}}$ \\
\hline IQF & $17.01 \pm 1.168^{\mathrm{B}}$ & $23.4 \pm 2.65^{\mathrm{A}}$ & $20.3 \pm 2.55^{\mathrm{AB}}$ \\
\hline $\mathrm{CF}$ & & $24.4 \pm 1.02^{\mathrm{A}}$ & $19.3 \pm 2.58^{\mathrm{AB}}$ \\
\hline \multicolumn{4}{|c|}{ Water holding capacity (\%) } \\
\hline NF & & $86.1 \pm 0.43^{\mathrm{AB}}$ & $82.4 \pm 10.22^{\mathrm{AB}}$ \\
\hline IQF & $89.33 \pm 1.150^{\mathrm{A}}$ & $83.2 \pm 2.01^{\mathrm{AB}}$ & $79.8 \pm 4.30^{\mathrm{B}}$ \\
\hline $\mathrm{CF}$ & & $85.4 \pm 1.73^{\mathrm{AB}}$ & $82.3 \pm 2.84^{\mathrm{AB}}$ \\
\hline
\end{tabular}

${ }^{1)}$ Freezing system: NF (natural convection freezing), IQF (individual quick freezing), and CF (cryogenic freezing).

${ }^{2)}$ Thawing system: NCT (natural convection thawing) and RT (running water thawing).

${ }^{\mathrm{A}-\mathrm{B}}$ Means with different superscripts within same parameters are significantly different $(p<0.05)$.

higher than that of the control $(p<0.05)$. There was no significant difference in the cooking loss among the treatments. Previous reports show that cooking loss does not differ between fresh and frozen meat (Leygonie et al., 2011). According to Vieira et al. (2009), lipid melting and protein denaturation lead to the release of free as well as bound water, causing no difference in the cooking loss.

The WHC of the freezing/thawing treatments was not different from that of the fresh control, with the exception of the IQF/RT treatments, in which WHC was significantly lower than that of the control $(p<0.05)$. Although no significant difference in WHC among the treatments was observed, the IQF and RT methods appeared to decrease the WHC of the beef. The former can be attributed to surface drying, which caused the concentration of solutes on the meat surface and unfolding of the meat proteins. Meanwhile, the latter could be due to the relatively high temperature achieved during thawing. Ambrosiadis et al. (1994) postulated that frozen meat thawed at colder temperatures has better WHC, even if the thawing process is slow. Taken together, our results demonstrate that both IQF and RT tend to reduce the water binding properties of meat. However, the values of the water binding parameters of these treatments were not evaluated as deterioration, because of the insignificance of the results.

\section{Instrumental color}

The CIE color parameters of the frozen/thawed beef are enlisted in Table 2. Frozen beef thawed by NCT had sig- 
Table 2. Effects of freezing and thawing methods on the CIE color of beef

\begin{tabular}{|c|c|c|c|c|c|}
\hline $\begin{array}{l}\begin{array}{l}\text { Freezing } \\
\text { system }^{1)}\end{array} \\
\end{array}$ & $\begin{array}{l}\text { Thawing } \\
\text { system }^{2)}\end{array}$ & $L^{*}$ & $a^{*}$ & $b^{*}$ & Total color difference $(\Delta E)$ \\
\hline \multicolumn{2}{|c|}{ Control $^{3)}$} & $41.2 \pm 2.53^{\mathrm{A}, 4)}$ & $18.3 \pm 1.53^{\mathrm{AB}}$ & $7.9 \pm 2.074^{\mathrm{B}}$ & \\
\hline \multirow{2}{*}{ NF } & $\mathrm{NCT}$ & $38.3 \pm 2.17^{\mathrm{C}}$ & $18.7 \pm 1.70^{\AA}$ & $9.5 \pm 1.680^{\mathrm{AB}}$ & $4.33 \pm 1.37^{\mathrm{A}}$ \\
\hline & RT & $39.5 \pm 1.57^{\mathrm{ABC}}$ & $19.6 \pm 1.40^{\mathrm{A}}$ & $9.9 \pm 1.671^{\mathrm{A}}$ & $3.62 \pm 1.41^{\mathrm{AB}}$ \\
\hline \multirow{2}{*}{ IQF } & NCT & $39.2 \pm 2.14^{\mathrm{BC}}$ & $16.9 \pm 0.90^{\mathrm{B}}$ & $9.2 \pm 0.777^{\mathrm{AB}}$ & $3.45 \pm 1.00^{\mathrm{AB}}$ \\
\hline & RT & $40.0 \pm 1.06^{\mathrm{ABC}}$ & $19.2 \pm 1.54^{\mathrm{A}}$ & $10.1 \pm 2.203^{\mathrm{A}}$ & $3.60 \pm 1.24^{\mathrm{AB}}$ \\
\hline \multirow{2}{*}{$\mathrm{CF}$} & NCT & $40.6 \pm 1.45^{\mathrm{AB}}$ & $18.6 \pm 1.90^{\mathrm{A}}$ & $9.4 \pm 1.593^{\mathrm{AB}}$ & $2.75 \pm 1.68^{\mathrm{B}}$ \\
\hline & RT & $41.4 \pm 1.63^{\mathrm{A}}$ & $16.9 \pm 1.46^{\mathrm{B}}$ & $10.4 \pm 1.644^{\mathrm{A}}$ & $3.77 \pm 0.88^{\mathrm{AB}}$ \\
\hline
\end{tabular}

${ }^{10}$ Freezing system: NF (natural conventional freezing), IQF (individual quick freezing), and CF (cryogenic freezing)

${ }^{2)}$ Thawing system: NCT (natural convection thawing) and RT (running water thawing)

${ }^{3)}$ Control, raw beef

${ }^{\text {A-C }}$ Means with different superscripts within same column are significantly different $(p<0.05)$.

nificantly lower $L^{*}$ value than that of the control $(p<$ 0.05 ). Alternately, the $L^{*}$ value for all the freezing treatments that were followed by RT was not different from the fresh control. No significant difference in the redness $\left(a^{*}\right)$ was detected between the treated beef samples and the fresh control. Among the treatments, samples with IQF/NCT and CF/RT had significantly lower $a^{*}$ value than the others $(p<0.05)$. Meanwhile, the $b^{*}$ value obtained by the freezing/thawing treatments tended to be higher than that of the fresh control, and all the samples thawed by RT exhibited higher $b^{*}$ value than that of the control $(p<$ 0.05 ). The color of meat depends on several factors such as the concentration and chemical status of the meat pigment protein, myoglobin $(\mathrm{Mb})$, the moisture content, and WHC (Farouk et al., 2004; Hong et al., 2005; Leygonie et al., 2011). Generally, frozen meat loses moisture during thawing; hence, the color of frozen/thawed meat is paler than that of the fresh control (Chwastowska-Siwiecka et al., 2013). Lipid oxidation during freezing and/or storage also affects meat discoloration, by directly influencing the redox states of $\mathrm{Mb}$ (Love and Pearson, 1971). Lipid oxidation also causes meat discoloration by promoting protein oxidation, leading to the moisture loss (Leygonie et al., 2011). Although the treatments in this study did not cause any remarkable change in the overall meat color, the color parameters reflect that rapid freezing and thawing at chilled temperatures is favorable since it minimizes meat discoloration. Based on $\Delta E$, significant difference in color was obtained only between NF/RT and $\mathrm{CF} / \mathrm{RT}(p<0.05)$. Thus, rapid freezing and thawing processes can minimize meat discoloration and it is recommended that thawing temperatures should be as low as possible.

\section{Shear force}

To evaluate the impact of the freezing and thawing pro- cedures on the tenderness of beef, shear forces of the beef samples were compared (Fig. 3). All the freezing/thawing treatments, except $\mathrm{CF} / \mathrm{RT}$, exhibited high shear forces in the range of 5.0-5.8 kg, compared to $4.8 \mathrm{~kg}$ in the fresh control $(p<0.05)$. Among the freezing treatments, no tendency in shear force was observed, but the meat looked tender following the IQF/NCT and CF/RT treatments. Although frozen/thawed meat is known to be less tender than fresh controls (Leygonie et al., 2011), the comparison of tenderness between the different freezing treatments is not consistent. Some studies suggest that frozen meat is tender compared to the fresh control. In this case, tissue damage that occurs due to ice crystal formation liberates proteases, which accelerate the rate of aging of the meat (Gambuteanu et al., 2013; Shanks et al., 2002; Vieira et al., 2009). Meanwhile, shear forces of meat are also affected by its moisture content. Hong et al. (2005) show that frozen/thawed meat with a higher drip loss (thawing

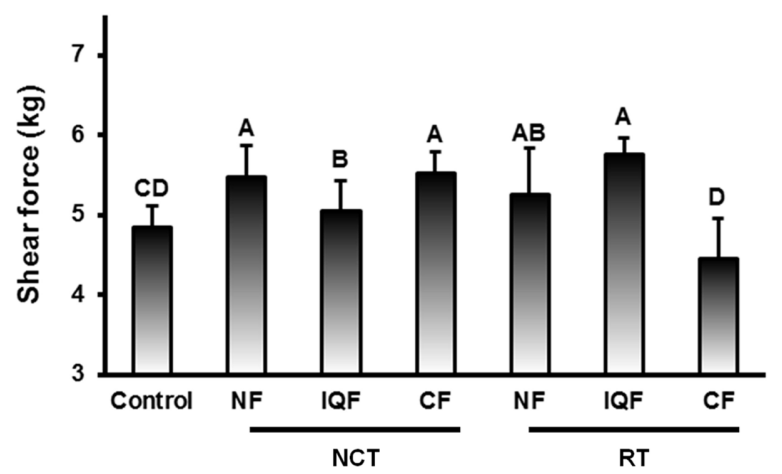

Fig. 3. Effects of the freezing and thawing methods on the shear force of beef samples. The errors bars indicate standard deviation ( $\mathrm{n}=3$, each repeated 16 times). Bars with different letters are significantly different $(p<0.05)$. The Y-axis denotes treatments including natural convection freezing (NF), individual quick freezing (IQF), cryogenic freezing $(\mathrm{CF})$, natural convection thawing (NCT), and running water thawing (RT). 


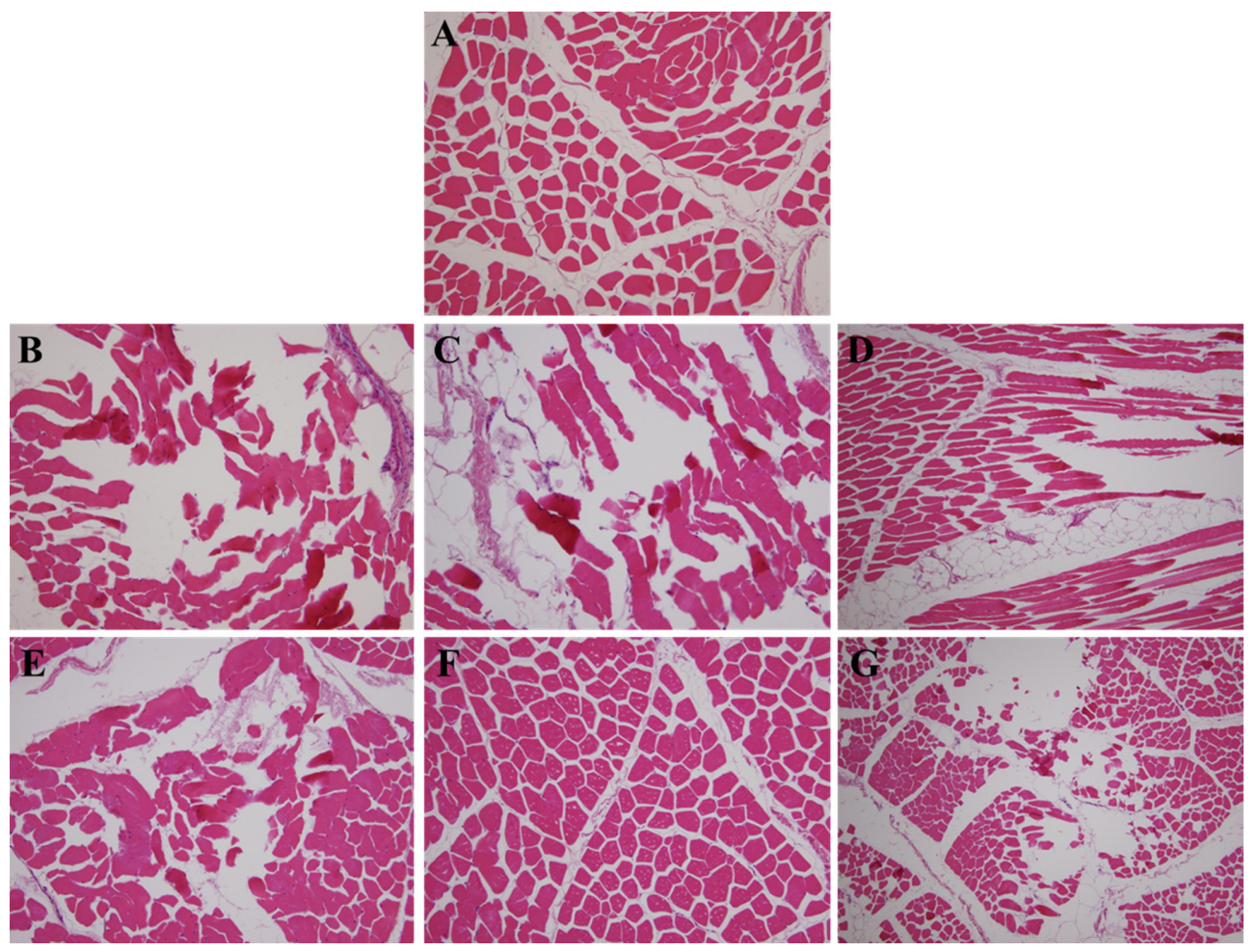

Fig. 4. Histological appearance of the frozen and thawed beef samples following the different freezing and thawing processes. (A) Control (raw beef), (B, E) natural convection freezing, (C, F) individual quick freezing, (D, G) cryogenic freezing, (B, C, D) natural convection thawing, and $(\mathrm{E}, \mathrm{F}, \mathrm{G})$ running water thawing.

and cooking losses) exhibits tougher texture. Such inconsistency in the results possibly reflects that the impact of freezing/thawing on meat tenderness is based on the period of aging. Crouse and Koohmaraie (1990) noted that beef aged after freezing is tender than that aged before freezing. Thus, the low beef tenderness caused by the freezing/ thawing treatments in this study can be attributed to the short aging period (freezing at $2 \mathrm{~d}$ post mortem).

\section{Light microscopy}

The light microscopy images of the fresh and frozen beef tissue are shown in Fig. 4. For the fresh control, transverse sections of the myofibrils were mostly homogeneous and the myofibrils were found to maintain their integrity. Meanwhile, segmental muscle and segmental coagulation necrosis were observed in the frozen/thawed meat, particularly after the IQF/NRT and NF/RT treatments. This phenomenon may result from ice crystal formation and recrystallization. Alternately, CF maintained the condition of myofibrils, although their density was not as intense as that of the fresh control. Although our results demonstrate that tissue damage during the freezing/ thawing processes is inevitable, we propose that the impact of tissue damage on meat quality is not remarkable when efficient freezing and thawing processes are applied.

\section{Conclusions}

The present study investigated the effects of the combination of various freezing and thawing techniques on the quality of beef. Although rapid freezing and thawing did not lead to a remarkable change in the overall beef quality, rapid freezing combined with chilled thawing appeared to minimize setbacks caused by the freezing/thawing cycle. For practical applications, IQF combined with NCT may be advantageous, if surface drying during IQF can be effectively prevented.

\section{Acknowledgements}

This work was carried out with the "Research Program for Agriculture Science \& Technology Development (Project No. PJ009440)", Rural Development Administration, Republic of Korea.

\section{References}

1. Ambrosiadis, I., Theodorakakos, N., Georgakis, S., and Lekas, 
S. (1994) Influence ofthawing methods on the quality of frozen meat and drip loss. Fleischwirtschaft 74, 284-286.

2. Carse, W. A. and Locker, R. H. (1974) A survey of $\mathrm{pH}$ values at the surface of beef and lamb carcasses, stored in a chiller. J. Sci. Food Agric. 25, 1529-1535.

3. Carson, J. K. (2006) Review of effective thermal conductivity models for foods. Int. J. Refrig. 29, 958-967.

4. Chwastowska-Siwiecka, I., Kondratowicz, J., Gugolek, A., and Matusevicius, P. (2013) Changes in the physicochemical properties of deep-frozen rabbit meat as dependent on thawing method. Vet. Med. Zoot. 62, 68-72.

5. Crouse, J. D. and Koohmaraie, M. (1990) Effect of freezing of beef on subsequent postmortem aging and shear force. $J$. Food Sci. 55, 573-574.

6. Farouk, M. M., Wieliczko, K. J., and Merts, I. (2004) Ultrafast freezing and low storage temperatures are not necessary to maintain the functional properties of manufacturing beef. Meat Sci. 66, 171-179.

7. Fernández, P. P., Otero, L., Guignon, B., and Sanz, P. D. (2006) High-pressure shift freezing versus high-pressure assisted freezing: Effects on the microstructure of a food model. Food Hydrocolloid. 20, 510-522.

8. Gambuteanu, C., Borda, D., and Alexe, P. (2013) The effect of freezing and thawing on technological properties of meat: Review. J. Agro. Proc. Technol. 19, 88-93.

9. Hong, G. P., Park, S. H., Kim, J. Y., Lee, C. H., Lee, S., and Min, S. G. (2005) The effect of thawing rate on the physicochemical properties of frozen ostrich meat. Food Sci. Biotechnol. 14, 676-680.

10. Hong, G. P., Shim, K. B., Choi, M. J., and Min, S. G. (2009) Effects of air blast thawing combined with infrared radiation on physical properties of pork. Korean J. Food Sci. An. 29, 302-309.

11. Hu, Z. and Sun, D. W. (2001) Predicting local surface heat transfer coefficients by different turbulent $\kappa-\varepsilon$ models to simulate heat and moisture transfer during air-blast chilling. Int. J. Refrig. 24, 702-717.

12. James, D. W. (1968) The thermal diffusivity of ice and water between -40 and $+60^{\circ}$ C. J. Mater. Sci. 3, 540-543.

13. Leung, M., Ching, W. H., Leung, D. Y. C., and Lam, G. C. K. (2007) Fluid dynamics and heat transfer in cold water thawing. J. Food Eng. 78, 1221-1227.

14. Leygonie, C., Britz, T. J., and Hoffman, L. C. (2011) Oxidative stability of previously frozen ostrich $M$. iliofibularis packaged under different modified atmosphere conditions. Int. J. Food Sci. Technol. 46, 1171-1178.

15. Li, B. and Sun, D. W. (2002) Novel methods for rapid freezing and thawing of foods-A review. J. Food Eng. 54, 175-182.

16. Love, J. D. and Pearson, A. M. (1971) Lipid oxidation in meat and meat products-A review. J. Am. Oil Chem. Soc. 48, 547549.

17. Meersman, F., Smeller, L., and Heremans, K. (2006) Protein stability and dynamics in the pressure-temperature plane. Biochim. Biophys. Acta. 1764, 346-354.

18. Shanks, B. C., Shanks, B. C., Wulf, D. M., and Maddock, R. J. (2002) The effect of freezing on Warner-Bratzler shear force values of beef longissimus steaksacross several post-mortem aging periods. J. Anim. Sci. 80, 2122-2125.

19. Vieira, C., Diaz, M.Y., Martinez, B., and Garcia-Cachan, M. D. (2009) Effect of frozen storage conditions (temperatureand length of storage) on microbial and sensoryquality of rustic crossbred beef at different stages ofaging. Meat Sci. 83, 398-404.

20. Wagner, J. R. and Aňón, M. C. (1985) Effect of freezing rate on the denaturation of myofibrillar proteins. J. Food Technol. 20, 735-744.

(Received 2014.9.16/Revised 2014.10.4/Accepted 2014.10.13) 